\title{
Study on Band-notched Charateristic Inheritance in UWB MIMO Antenna Array Design
}

\author{
Yuchen Zhao*, Lu Wang, Dandan Wang and Xiaoli Xi \\ Xi'an University of Technology, Xi'an, 710048, China \\ ${ }^{*}$ Corresponding author
}

\begin{abstract}
In this paper, the band-notched characteristic inheritance in ultra-wide band (UWB) multiple input multiple output (MIMO) antenna array design is studied. First, the structure parameters and return loss of a monopole antenna are optimized to obtain desired UWB property. Then, the bandnotched characteristic is realized in $7.9-8.4 \mathrm{GHz}$ by using the rectangle split ring resonator (r-SRR). Furthermore, this UWB antenna is utilized to form a MIMO antenna array based on quasi-self-complementary antenna technique. Finally, the simulation results show that on the one hand, the band-notched property of the unit antenna can be inherited by the antenna array; on the other hand, the bandwidth of the stop band for antenna array is increased by about $66 \%$ compared with that of the antenna unit.
\end{abstract}

Keywords- UWB antenna; MIMO antenna array; band-notched characteristic; inheritance

\section{INTRODUCTION}

With the rising and rapid development of the wireless communication, the increasing demands are moving towards large channel capacity, high transmission speed and stable mobile radio link. In order to adapt to this tendency, many novel techniques are developed in the past few decades. Among which, ultra-wide band (UWB) technique and multiple input multiple output (MIMO) concept have aroused wide interest in both academia and industry ${ }^{[1-2]}$. Hence, as one of the most important component in the new generation wireless communication system, the UWB antenna and the MIMO antenna have evolved so quickly and become one of the hottest field in the forefront of antenna design.

For any UWB antenna, there is always a great possibility that its operation frequency band will overlap with that of many other wireless systems, which might lead to undesired interference between different devices. To overcome this problem, the band-notched characteristic, which can achieve band-stop property in a particular frequency band to filter transmission signal from interference system directly, has been introduced to UWB antenna. Another major problem for a UWB system is that the limited output power makes it difficult to achieve long-distance transmission. Since MIMO technology can decrease channel fading and improve the transmission quality of the mobile terminals, the combination of UWB characteristic and MIMO property can further improve the performance of the wireless devices. Based on the above analysis, it is reasonable to conclude that the design of UWB MIMO antenna array with band-notched characteristic is of significance in both theoretical research and practical engineering.

Until now, many scientists and engineers have devoted themselves to the studies of UWB antenna, UWB antenna with band-notched characteristic and MIMO antenna array ${ }^{[3-4]}$. According to these studies, the general design process for UWB MIMO antenna array with band-notched characteristic can be divided into four steps: UWB antenna unit design; bandnotched characteristic design for antenna unit; MIMO antenna array design; band-notched characteristic adjustment for antenna array. To the best of our knowledge, most of relative researches usually present their MIMO antenna array directly and rarely analyze how the MIMO array inherit the bandnotched characteristic from its UWB antenna unit. This is the starting point of this work.

Hence, the band-notched characteristic inheritance in UWB MIMO antenna array design is studied in this paper. First of all, a monopole antenna unit is optimized to obtain desired UWB property. Second, the rectangle split ring resonator (r-SRR) is used to realize the band-notched characteristic in $7.9-8.4 \mathrm{GHz}$ for the UWB antenna unit. Then, a UWB MIMO antenna array with four antenna unit is presented by using quasi-selfcomplementary antenna technique. Finally, the simulations discover that the antenna array can inherit band-notched characteristic directly from its antenna unit and the main change is the increase in stop band bandwidth. Specifically, the stop band width of the array is increased by about $66 \%$ compared with that of the unit here.

\section{SimULATION AND ANALYSIS}

\section{A. UWB Antenna Unit}

The geometry of the UWB antenna unit, as shown in Fig.1, has one planar monopole element ${ }^{[5]}$. The three-dimensional size of this antenna is $25 \mathrm{~mm} \times 25 \mathrm{~mm} \times 1.6 \mathrm{~mm}$. The substrate is FR4 with relative permittivity 4.6 and loss tangent 0.019 . According to the reference, the $S_{11}$ of this UWB antenna unit is very close to $-10 \mathrm{~dB}$ at $4 \mathrm{GHz}$ frequency point. Hence, the structure parameters are optimized to obtain better return loss property. The optimized geometry parameters are $\mathrm{L}=25 \mathrm{~mm}, \mathrm{~L}_{1}=3 \mathrm{~mm}$, $\mathrm{L}_{2}=3 \mathrm{~mm}, \mathrm{~L}_{3}=2.5 \mathrm{~mm}, \mathrm{~L}_{4}=2 \mathrm{~mm}, \mathrm{~L}_{5}=6 \mathrm{~mm}, \mathrm{~L}_{6}=3 \mathrm{~mm}, \mathrm{~L}_{7}=1 \mathrm{~mm}$, $\mathrm{L}_{8}=3 \mathrm{~mm}, \quad \mathrm{~L}_{9}=6 \mathrm{~mm}, \mathrm{~W}=25 \mathrm{~mm}, \mathrm{~W}_{1}=2 \mathrm{~mm}, \mathrm{~W}_{2}=11 \mathrm{~mm}$, $\mathrm{W}_{3}=3 \mathrm{~mm}, \mathrm{~W}_{4}=2 \mathrm{~mm}, \mathrm{~W}_{5}=8.8 \mathrm{~mm}, \mathrm{~W}_{7}=5 \mathrm{~mm}, \mathrm{~W}_{8}=4 \mathrm{~mm}$, $\mathrm{W}_{9}=7.8 \mathrm{~mm}$. Fig. 2 displays the comparison between the $S_{11}$ curves before and after optimization. It can be found that the return loss property satisfies the Federal Communications 
Commission requirement in the whole $3.1-10.6 \mathrm{GHz}$ frequency band and the $S_{11}$ value in $4 \mathrm{GHz}$ frequency point decreases from $-9.8 \mathrm{~dB}$ to $-10.9 \mathrm{~dB}$.

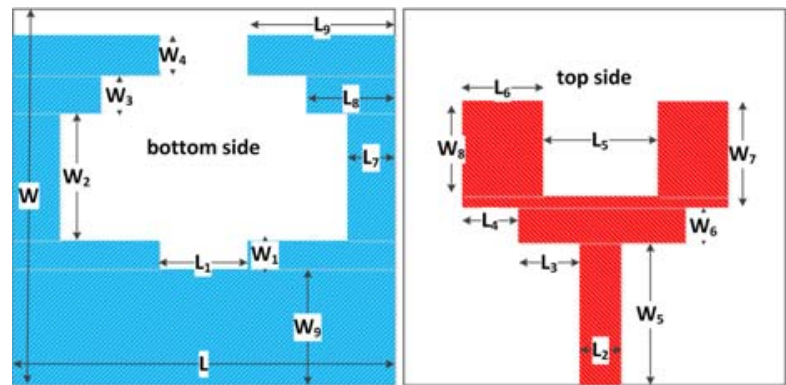

FIGURE I. GEOMETRY OF UWB ANTENNA UNIT

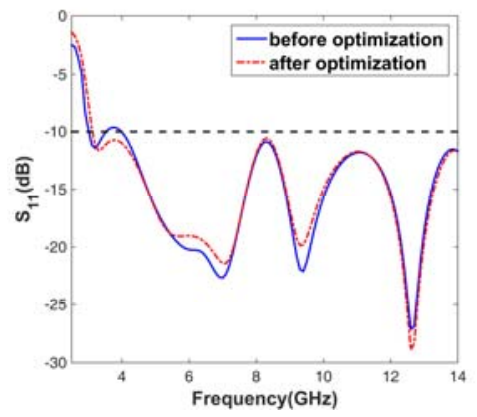

FIGURE II. DIFFERENT S $S_{11}$ CURVES

\section{B. Band-notched Characteristic}

The r-SRR is used here to generate band-notched characteristic for UWB antenna, as shown in Fig.3. The stop band design objective is chosen to be in the uplink frequency band for X-band satellite communication, i.e., 7.9-8.4GHz.

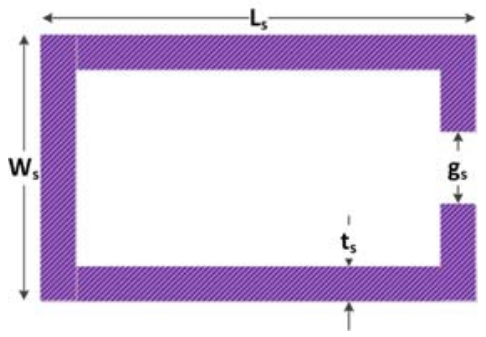

FIGURE III. STRUCTUE OF r-SRR

The resonant frequency $f_{\text {res }}$ of this resonator can be estimated approximately by the following expression

$$
2\left(L_{s}+W_{s}-2 t_{s}\right)=\frac{c}{2 f_{\text {res }} \sqrt{\varepsilon_{\text {eff }}}}
$$

where

$$
\varepsilon_{\text {eff }}=\frac{\varepsilon_{r}+1}{2}+\frac{\varepsilon_{r}-1}{2}\left(1+\frac{12 h}{w_{f}}\right)^{-0.5}
$$

here $\varepsilon$ is the relative permittivity of the substrate, $h$ is the height of the substrate and $w_{f}$ is the width of the feed line.

Two resonant rings are placed on both sides of the feed line to achieve desired band-notched characteristic. The geometry parameters of each resonator are $\mathrm{L}_{\mathrm{s}}=5 \mathrm{~mm}, \mathrm{~W}_{\mathrm{s}}=2.4 \mathrm{~mm}$, $\mathrm{g}_{\mathrm{s}}=0.8 \mathrm{~mm}, \mathrm{t}_{\mathrm{s}}=0.3 \mathrm{~mm}$. At the same time, an antenna structure parameter is also adjusted to obtain better return loss property, i.e., $L_{9}=5 \mathrm{~mm}$ here. Fig. 4 shows the obtained $\mathrm{S}_{11}$ curves.

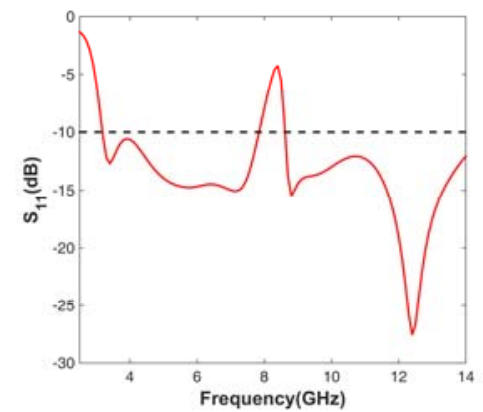

FIGURE IV. BAND-NOTCHED CHARACTERISTIC

As shown in the figure, the band-notched characteristic can be found in the $7.9-8.5 \mathrm{GHz}$, which is basically consistent with the design objective. The bandwidth of the stop band is about $0.6 \mathrm{GHz}$.

\section{MIMO Antenna Array with Band-notched Characteristic}

Based on the quasi-self-complementary antenna technique $^{[5-6]}$, a MIMO antenna array with band-notched characteristic is presented. The layout of this UWB MIMO antenna array, which is illustrated in Fig.5, includes four antenna units. Each unit has a 7.9-8.5GHz stop band generated by the application of r-SRR. The whole size of the MIMO array is $50 \mathrm{~mm} \times 50 \mathrm{~mm} \times 1.6 \mathrm{~mm}$.

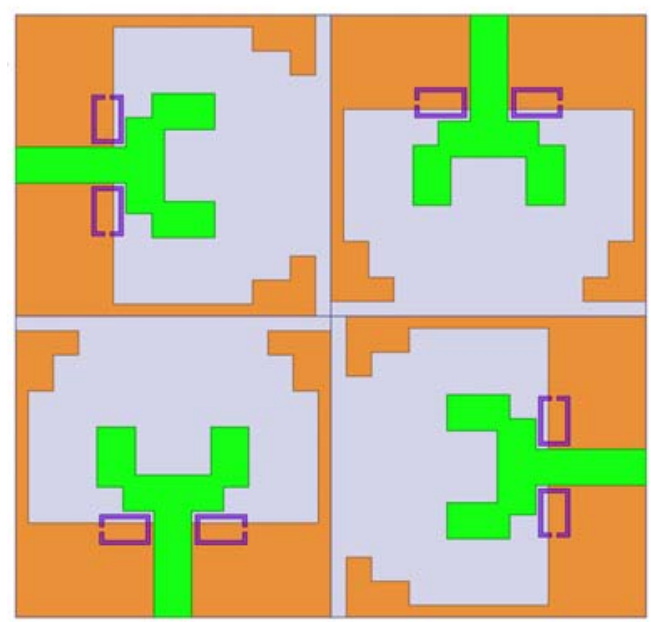

FIGURE V. LAYOUT OF UWB MIMO ANTENNA ARRAY

For convenience, the UWB antenna in the top left is denoted as unit 1 ; the top right antenna is denoted as unit 2; the 
bottom left antenna is unit 3; the bottom right antenna is unit 4 . The orientation of each unit is orthogonal to each other.

The correlation coefficient is a key parameter to determine whether MIMO antenna can be applied to MIMO system. For two antenna unites, for example, unit 1 and unit 2, their correlation coefficient is given by

$$
\rho=\frac{\left|S_{11}^{*} S_{12}+S_{11}^{*} S_{12}\right|^{2}}{\left(1-\left|S_{11}\right|^{2}-\left|S_{21}\right|^{2}\right)\left(1-\left|S_{22}\right|^{2}-\left|S_{12}\right|^{2}\right)}
$$

Fig.6 gives the calculated correlation coefficients for the presented MIMO antenna array.

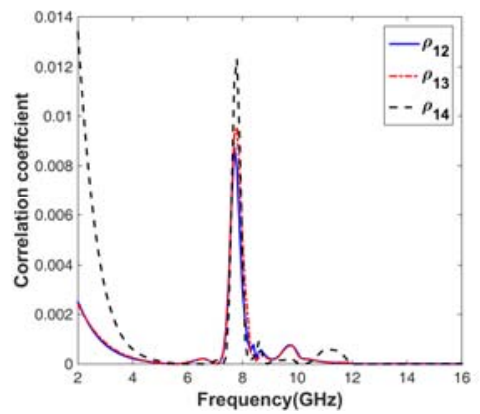

FIGURE VI. CALCULATED CORRELATION COEFFCIENTS

According to the figure, the correlation coefficients between different antenna units are less than 0.014 in the whole UWB operation frequency band. Since the correlation coefficient should not be greater than 0.5 in MIMO technique, this demonstrates the rationality of the presented MIMO antenna array.

Last but not least, the band-notched property inheritance is studied in Fig.7. It is obvious that both the UWB property and the band-notched characteristic of the antenna unit is inherited by the MIMO antenna array. What's more, it is worth noting that the stop band is $7.5-8.5 \mathrm{GHz}$ for the MIMO antenna array, i.e., the bandwidth is increased by about $66 \%$ compared with that of the antenna unit. Hence, when designing an UWB MIMO antenna array with band-notched characteristic, one needs to consider the inheritance and variability of the stop band. Otherwise, some useful signals might be rejected by the UWB MIMO system.

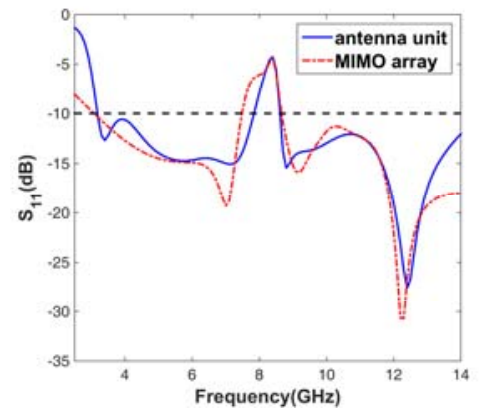

FIGURE VII. BAND-NOTCHED PROPERTY INHERITANCE

\section{CONCLUSIONS}

In summary, the band-notched characteristic inheritance in UWB MIMO antenna array design is studied in this paper. Through four design steps, we present a UWB MIMO antenna array with band-notched characteristic. The simulation results show that the stop band width is $0.6 \mathrm{GHz}$ for UWB antenna unit and $1.0 \mathrm{GHz}$ for MIMO antenna array, which means that the bandwidth is increased by about $66 \%$. Such phenomenon suggests us to consider the band-notched characteristic inheritance and variability in this four-step design process.

\section{ACKNOWLEDGMENT}

This work was partly supported by the National Natural Science Foundation of China (No.61501369), the Postdoctoral Foundation (No.2016M592904XB), the Foundations of Xi'an University of Technology (No.105-211423, No.2016CX040, No.310-252051618).

\section{REFERENCES}

[1] G. Srivastava and A. Mohan, "Compact MIMO Slot Antenna for UWB Applications,” IEEE Antennas and Wireless Propagation letters, vol. 15, no. 1, pp. 1057-1060, March 2015.

[2] D. Manteuffel and R. Martens, "Compact Multimode Multielement Antenna for Indoor UWB Massive MIMO," IEEE Transactions on Antennas and Propagation, vol. 64, no. 7, pp. 2689 - 2697, July 2016.

[3] J. F. Li, Q. X. Chu, Z. H. Li and X. X. Xia, "Compact Dual BandNotched UWB MIMO Antenna With High Isolation,” IEEE Transactions on Antennas and Propagation, vol. 61, no. 9, pp. 4759 4766, September 2013

[4] L. Liu, S. W. Cheung and T. I. Yuk, "Compact MIMO Antenna for Portable UWB Applications With Band-Notched Characteristic,” IEEE Transactions on Antennas and Propagation, vol. 63, no. 5, pp. 1917-1924, May 2015.

[5] J. B. Zhao, "Design of miniaturized MIMO antenna with low mutual coupling,” Chengdu: University of Electronic Science and Technology of China, June 2015 (in Chinese)

[6] X. L. Liu, Z. D. Wang, Y. Z. Yin, J. Ren and J. J. Wu, “A compact ultrawideband MIMO antenna using QSCA for high isolation,” IEEE Antennas and Wireless Propagation letters, vol. 13, no. 1, pp. 1497-1500, April 2014. 